\title{
On sums of distinct representatives
}

\author{
by \\ Hui-Qin CaO and Zhi-Wei Sun (Nanjing)
}

1. Introduction. In combinatorics, for a finite sequence

$$
\left\{A_{i}\right\}_{i=1}^{n}
$$

of sets, a sequence

$$
\left\{a_{i}\right\}_{i=1}^{n}
$$

of elements is called a system of distinct representatives (abbreviated to SDR) of (1) if $a_{1} \in A_{1}, \ldots, a_{n} \in A_{n}$ and $a_{i} \neq a_{j}$ for all $1 \leq i<j \leq n$. A celebrated theorem of $\mathrm{P}$. Hall $[\mathrm{H}]$ says that (1) has an SDR if and only if

$$
\left|\bigcup_{i \in I} A_{i}\right| \geq|I| \quad \text { for all } I \subseteq\{1, \ldots, n\} .
$$

Clearly (1) has an SDR provided that $\left|A_{i}\right| \geq i$ for all $i=1, \ldots, n$, in particular an SDR of (1) exists if $\left|A_{1}\right|=\ldots=\left|A_{n}\right| \geq n$ or $0<\left|A_{1}\right|<\ldots$ $<\left|A_{n}\right|$.

Let $G$ be an additive abelian group and $A_{1}, \ldots, A_{n}$ its subsets. We associate any $\operatorname{SDR}(2)$ of (1) with the sum $\sum_{i=1}^{n} a_{i}$ and set

$$
\begin{aligned}
S\left(\left\{A_{i}\right\}_{i=1}^{n}\right) & =S\left(A_{1}, \ldots, A_{n}\right) \\
& =\left\{a_{1}+\ldots+a_{n}:\left\{a_{i}\right\}_{i=1}^{n} \text { forms an SDR of }\left\{A_{i}\right\}_{i=1}^{n}\right\} .
\end{aligned}
$$

Of course, $S\left(A_{1}, \ldots, A_{n}\right) \neq \emptyset$ if and only if (3) holds. A fascinating and challenging problem is to give a sharp lower bound for $\left|S\left(\left\{A_{i}\right\}_{i=1}^{n}\right)\right|$ and determine when the bound can be reached.

Let $p$ be a prime. In 1964 P. Erdős and H. Heilbronn (cf. [EH] and [G]) conjectured that for each nonempty subset $A$ of $\mathbb{Z}_{p}=\mathbb{Z} / p \mathbb{Z}$ there are at least $\min \{p, 2|A|-3\}$ elements of $\mathbb{Z}_{p}$ that can be written as the sum of two distinct elements of $A$. With the help of Grassmann spaces this was

1991 Mathematics Subject Classification: Primary 11B75; Secondary 05A05.

The research is supported by the Return-from-abroad Foundations of the Chinese Educational Committee and Nanjing City. 
confirmed by J. A. Dias da Silva and Y. O. Hamidoune [DH] in 1994, in fact they proved the following generalization for $n$-fold sums: If $A \subseteq \mathbb{Z}_{p}$ then

$$
\left|n^{\wedge} A\right| \geq \min \left\{p, n|A|-n^{2}+1\right\}
$$

where $n^{\wedge} A$ denotes the set of sums of $n$ distinct elements of $A$, i.e. $n^{\wedge} A=$ $S(A, \ldots, A)$ with $A$ repeated $n$ times on the right hand side. In 1995 and 1996, N. Alon, M. B. Nathanson and I. Z. Ruzsa [ANR1, ANR2] introduced an ingenious polynomial method and obtained the following result by contradiction: Let $F$ be any field of characteristic $p$ and $A_{1}, \ldots, A_{n}$ its subsets with $0<\left|A_{1}\right|<\ldots<\left|A_{n}\right|<\infty$. Then

$$
\left|S\left(A_{1}, \ldots, A_{n}\right)\right| \geq \min \left\{p, \sum_{i=1}^{n}\left|A_{i}\right|-\frac{n(n+1)}{2}+1\right\}
$$

providing $N<p$ where $N=\sum_{i=1}^{n}\left|A_{i}\right|-n(n+1) / 2$. We mention that (6) also holds in the case $N \geq p$. In fact, let $s$ be the smallest positive integer with $\sum_{i=1}^{s}\left(\left|A_{i}\right|-i\right)>N-p$ and choose $A_{1}^{\prime} \subseteq A_{1}, \ldots, A_{n}^{\prime} \subseteq A_{n}$ so that $\left|A_{i}^{\prime}\right|=i$ for $i<s, A_{i}^{\prime}=A_{i}$ for $i>s$, and

$$
\left|A_{s}^{\prime}\right|=s-1+\sum_{i=1}^{s}\left(\left|A_{i}\right|-i\right)-(N-p) \leq\left|A_{s}\right|-1 .
$$

Then $\left|A_{1}^{\prime}\right|<\ldots<\left|A_{n}^{\prime}\right|, N^{\prime}=\sum_{i=1}^{n}\left|A_{i}^{\prime}\right|-n(n+1) / 2=p-1$ and so

$$
\left|S\left(A_{1}, \ldots, A_{n}\right)\right| \geq\left|S\left(A_{1}^{\prime}, \ldots, A_{n}^{\prime}\right)\right| \geq N^{\prime}+1=p=\min \{p, N\} .
$$

Alon, Nathanson and Ruzsa [ANR2] posed the question when the lower bound in (6) can be reached and considered it to be interesting.

In view of the fundamental theorem on finitely generated abelian groups (cf. $[\mathrm{J}]$ ), if a finite addition theorem holds in $\mathbb{Z}$ then it holds in any torsionfree abelian groups. So, without any loss of generality, we may work within $\mathbb{Z}$.

For a finite subset $A$ of $\mathbb{Z}$, in 1995 Nathanson $[\mathrm{N}]$ showed the inequality $\left|n^{\wedge} A\right| \geq n|A|-n^{2}+1$ and proved that if equality holds then $A$ must be an arithmetic progression providing $2 \leq n<|A|-2$. The same result was independently obtained by Y. Bilu [B].

Let $A_{1}, \ldots, A_{n}$ be finite subsets of $\mathbb{Z}$ with $0<\left|A_{1}\right|<\ldots<\left|A_{n}\right|$. Take a sufficiently large prime $p$ greater than $\sum_{i=1}^{n}\left|A_{i}\right|-n(n+1) / 2$ and the largest element of $S\left(A_{1}, \ldots, A_{n}\right)$. Applying the Alon-Nathanson-Ruzsa result stated above, we have the inequality

$$
\left|S\left(A_{1}, \ldots, A_{n}\right)\right| \geq \sum_{i=1}^{n}\left|A_{i}\right|-\frac{n(n+1)}{2}+1=\sum_{i=1}^{n}\left(\left|A_{i}\right|-i\right)+1 .
$$

In this paper we will make a new approach to sums of distinct representatives. The method allows us to give a somewhat constructive proof of (7) provided that $A_{1}, \ldots, A_{n}$ are finite nonempty subsets of $\mathbb{Z}$ with distinct 
cardinalities. Furthermore we are able to make key progress in the equality case.

Let us first look at two examples.

ExAmple 1. Let $a \in \mathbb{Z}$ and $d \in \mathbb{Z} \backslash\{0\}$. Let $k \geq n \geq 1, A=\{a+j d$ : $j=0,1, \ldots, k-1\}$, and $A_{1}, \ldots, A_{n}$ be subsets of $A$ with $\left|A_{i}\right|=k-n+i$ for every $i=1, \ldots, n$. Obviously $S\left(A_{1}, \ldots, A_{n}\right) \subseteq n^{\wedge} A$. If $S \subseteq A$ and $|S|=n$, then for each $i=1, \ldots, n$ at least $i$ elements of $S$ lie in $A_{i}$ since $\left|S \backslash A_{i}\right| \leq$ $\left|A \backslash A_{i}\right|=n-i$, therefore we can write $S$ in the form $\left\{a_{1}, \ldots, a_{n}\right\}$ where $a_{1} \in A_{1}, \ldots, a_{n} \in A_{n}$. So $n^{\wedge} A \subseteq S\left(A_{1}, \ldots, A_{n}\right)$. Let $X=\left\{j_{1}+\ldots+j_{n}: 0 \leq\right.$ $\left.j_{1}<\ldots<j_{n}<k\right\}$. For each $j=0,1, \ldots, n(k-n)$, there exist $0 \leq u<n$ and $0 \leq v \leq k-n$ such that $j=u(k-n)+v$, hence

$$
\begin{aligned}
\frac{n(n-1)}{2}+j & =\sum_{i=1}^{n}(i-1)+u(k-n)+v \\
& =\sum_{0<i<n-u}(i-1)+(n-u-1+v)+\sum_{n-u<i \leq n}(k-n+i-1)
\end{aligned}
$$

belongs to $X$. Thus $\{n(n-1) / 2+j: 0 \leq j \leq n(k-n)\} \subseteq X$. Apparently the least and the largest elements of $X$ are $0+1+\ldots+(n-1)=n(n-1) / 2$ and $(k-n)+\ldots+(k-1)=n(n-1) / 2+n(k-n)$ respectively. So, by the above

$$
\begin{aligned}
S\left(A_{1}, \ldots, A_{n}\right) & =n^{\wedge} A=\left\{\sum_{i=1}^{n}\left(a+j_{i} d\right): 0 \leq j_{1}<\ldots<j_{n}<k\right\} \\
& =\{n a+x d: x \in X\} \\
& =\left\{n a+\left(\frac{n(n-1)}{2}+j\right) d: 0 \leq j \leq n(k-n)\right\}
\end{aligned}
$$

and hence

$$
\left|S\left(A_{1}, \ldots, A_{n}\right)\right|=\left|n^{\wedge} A\right|=n(|A|-n)+1=\sum_{i=1}^{n}\left|A_{i}\right|-\frac{n(n+1)}{2}+1 .
$$

EXAMPLE 2 (cf. [N]). Let $a_{0}, a_{1}, a_{2}, a_{3} \in \mathbb{Z}, a_{0}<a_{1}<a_{2}<a_{3}$ and $a_{3}-a_{2}=a_{1}-a_{0}$ (but $a_{2}-a_{1}$ may be different from $a_{1}-a_{0}$ ). Let $A_{1}=$ $\left\{a_{0}, a_{1}, a_{2}\right\}$ and $A_{2}=\left\{a_{0}, a_{1}, a_{2}, a_{3}\right\}$. Then

$$
S\left(A_{1}, A_{2}\right)=\left\{a_{0}+a_{1}, a_{0}+a_{2}, a_{0}+a_{3}=a_{1}+a_{2}, a_{1}+a_{3}, a_{2}+a_{3}\right\} .
$$

Note that in this example $\left|A_{1}\right|=3<\left|A_{2}\right|=4<\left|S\left(A_{1}, A_{2}\right)\right|=5=$ $\left|A_{1}\right|+\left|A_{2}\right|-2(2+1) / 2+1$.

Now we introduce some notations to be used throughout the paper. For a subset $A$ of $\mathbb{Z},-A$ refers to $\{-a: a \in A\}, \min A$ and $\max A$ denote the least and the largest elements of $A$ respectively. If there exist $a \in \mathbb{Z}, d \in \mathbb{Z} \backslash\{0\}$ 
and a positive integer $k$ such that

$$
A=\{a+j d: 0 \leq j<k\},
$$

then we call $A$ an arithmetic progression (for short, AP).

In this paper, by a novel method we obtain the following

Theorem. Let $A_{1}, \ldots, A_{n}$ be subsets of $\mathbb{Z}$ with $0<\left|A_{1}\right|<\ldots<$ $\left|A_{n}\right|<\infty$. Then inequality (7) holds. Moreover, in the equality case we have $\bigcup_{i=1}^{m} A_{i}=A_{m}$ for every $m \in M=\left\{1 \leq j<n:\left|A_{j+1}\right|>\left|A_{j}\right|+1\right\} \cup\{n\}$, and $A_{n}$ forms an $A P$ unless $n=1$ or $\left|A_{1}\right| \leq 3$.

The result of Nathanson and Bilu stated above actually follows from the Theorem. For $i=1, \ldots, n$ let $A_{i} \subseteq A$ and $\left|A_{i}\right|=|A|-(n-i)$. Obviously $0<\left|A_{1}\right|<\ldots<\left|A_{n}\right|<\infty$. It follows from Example 1 and the Theorem that

$$
\left|n^{\wedge} A\right|=\left|S\left(A_{1}, \ldots, A_{n}\right)\right| \geq \sum_{i=1}^{n}\left|A_{i}\right|-\frac{n(n+1)}{2}+1=n|A|-n^{2}+1 .
$$

When $\left|n^{\wedge} A\right|=n|A|-n^{2}+1$, we have

$$
\left|S\left(A_{1}, \ldots, A_{n}\right)\right|=\sum_{i=1}^{n}\left|A_{i}\right|-\frac{n(n+1)}{2}+1,
$$

hence by the Theorem if $2 \leq n \leq|A|-3$ (i.e., $n \geq 2$ and $\left|A_{1}\right| \geq 4$ ) then $A=A_{n}$ is an AP.

In the next section we shall provide two lemmas. The proof of the Theorem will be given in Section 3 .

\section{Auxiliary results}

Lemma 1. Let $G$ be an additive abelian group, and $A_{1}, \ldots, A_{n}$ its $f$ nite subsets. Let $r \in\{1, \ldots, n\}$ and suppose that $\left\{a_{i}\right\}_{i \neq r}$ forms an $S D R$ of $\left\{A_{i}\right\}_{i \neq r}$. Then

(i) There exists a $J \subseteq\{1, \ldots, n\}$ containing $r$ such that if $J \subseteq I \subseteq$ $\{1, \ldots, n\}$ then

$$
S_{r}^{(I)}\left(\bigcup_{j \in J} A_{j}\right) \subseteq S\left(\left\{A_{i}\right\}_{i \in I}\right), \quad\left\{i \in I \backslash\{r\}: a_{i} \in \bigcup_{j \in J} A_{j}\right\}=J \backslash\{r\}
$$

and hence

$$
\left|S_{r}^{(I)}\left(\bigcup_{j \in J} A_{j}\right)\right|=\left|\bigcup_{j \in J} A_{j}\right|-|J|+1,
$$

where for any subset $A$ of $G$ we let

$$
S_{r}^{(I)}(A)=\left\{\sum_{i \in I} a_{i}: a_{r} \in A \backslash\left\{a_{i}: i \in I \backslash\{r\}\right\}\right\} .
$$


(ii) Let $k_{r}=\left|A_{r}\right|<\ldots<k_{n}=\left|A_{n}\right|$. For any $J$ described in (i) and $I \subseteq\{1, \ldots, n\}$ containing $J$, we have

$$
\left|S_{r}^{(I)}\left(\bigcup_{j \in J} A_{j}\right)\right| \geq k_{r}-r+1,
$$

and equality holds if and only if there exists an $l \in\{r, \ldots, n\}$ for which $J=\{1, \ldots, l\}, \bigcup_{j=1}^{l} A_{j}=A_{l}$ and $k_{l}=k_{r}+(l-r)$.

Proof. (i) Let $\mathcal{J}$ be the class of those $J \subseteq\{1, \ldots, n\}$ containing $r$ such that if $J \subseteq I \subseteq\{1, \ldots, n\}$ then for each $j \in J$ there exists a oneto-one mapping $\sigma_{I, j}: I \backslash\{r\} \rightarrow I \backslash\{j\}$ for which $a_{i} \in A_{\sigma_{I, j}(i)}$ for all $i \in I \backslash\{r\}$. Obviously $\mathcal{J}$ is nonempty (for, $\{r\}$ belongs to $\mathcal{J}$ ) and finite. Let $J$ be any maximal set in $\mathcal{J}$ with respect to the semiorder $\subseteq$, and let $J \subseteq I \subseteq\{1, \ldots, n\}$.

Set $A=\bigcup_{j \in J} A_{j}$. Apparently $J^{\prime}=\{r\} \cup\left\{i \in I \backslash\{r\}: a_{i} \in A\right\}$ contains $J$. Let $J^{\prime} \subseteq I^{\prime} \subseteq\{1, \ldots, n\}$. Since $J \in \mathcal{J}$ and $J \subseteq I^{\prime}$, for $j \in J$ there is a one-to-one mapping $\sigma_{I^{\prime}, j}: I^{\prime} \backslash\{r\} \rightarrow I^{\prime} \backslash\{j\}$ such that $a_{i} \in A_{\sigma_{I^{\prime}, j}(i)}$ for all $i \in I^{\prime} \backslash\{r\}$. For $j^{\prime} \in J^{\prime} \backslash J$, there is a $j \in J$ with $a_{j^{\prime}} \in A_{j}$. Since $J \in \mathcal{J}$ and $I^{\prime \prime}=I^{\prime} \backslash\left\{j^{\prime}\right\} \supseteq J$, there also exists a one-to-one mapping $\sigma_{I^{\prime \prime}, j}: I^{\prime \prime} \backslash\{r\} \rightarrow I^{\prime \prime} \backslash\{j\}$ such that $a_{i} \in A_{\sigma_{I^{\prime \prime}, j}(i)}$ for $i \in I^{\prime \prime} \backslash\{r\}$. Obviously by letting $j^{\prime} \in I^{\prime} \backslash\{r\}$ correspond to $j \in I^{\prime} \backslash\left\{j^{\prime}\right\}$ we can extend $\sigma_{I^{\prime \prime}, j}$ to a one-to-one mapping $\sigma_{I^{\prime}, j^{\prime}}: I^{\prime} \backslash\{r\} \rightarrow I^{\prime} \backslash\left\{j^{\prime}\right\}$ for which $a_{i} \in A_{\sigma_{I^{\prime}, j^{\prime}}(i)}$ for all $i \in I^{\prime} \backslash\{r\}$. Thus $J^{\prime} \in \mathcal{J}$. As $J \subseteq J^{\prime}$ and $J$ is a maximal set in $\mathcal{J}$, we must have $J^{\prime}=J$, i.e., $\left\{i \in I \backslash\{r\}: a_{i} \in \bigcup_{j \in J} A_{j}\right\}=J \backslash\{r\}$.

If $j \in J$ and $x_{j} \in A_{j} \backslash\left\{a_{i}: i \in I \backslash\{r\}\right\}$, then $x_{j}+\sum_{i \in I \backslash\{r\}} a_{i} \in$ $S\left(\left\{A_{i}\right\}_{i \in I}\right)$ because $a_{i} \in A_{\sigma_{I, j}(i)}$ for $i \in I \backslash\{r\}$. So $S_{r}^{(I)}(A) \subseteq S\left(\left\{A_{i}\right\}_{i \in I}\right)$. Note that

$$
\begin{aligned}
\left|S_{r}^{(I)}(A)\right| & =\left|A \backslash\left\{a_{i}: i \in I \backslash\{r\}\right\}\right|=|A|-\left|\left\{i \in I \backslash\{r\}: a_{i} \in A\right\}\right| \\
& =|A|-|J \backslash\{r\}|=|A|-|J|+1
\end{aligned}
$$

This proves part (i).

(ii) Let $J$ be as described in (i), $A=\bigcup_{j \in J} A_{j}$ and $J \subseteq I \subseteq\{1, \ldots, n\}$. If $|J|<r$, then

$$
|A|-|J| \geq\left|A_{r}\right|-|J|>k_{r}-r .
$$

When $|J| \geq r$, clearly $\max J \geq r$ and $k_{\max J}-k_{r}=\sum_{r<i \leq \max J}\left(k_{i}-k_{i-1}\right) \geq$ $\max J-r$, therefore

$$
|A|-|J| \geq\left|A_{\max } J\right|-|J| \geq k_{r}+\max J-r-|J| \geq k_{r}-r,
$$

and $|A|-|J|=k_{r}-r$ if and only if

$$
A=A_{\max J}, \quad k_{\max J}=k_{r}+\max J-r, \quad \max J=|J|,
$$


i.e.,

$$
J=\{1, \ldots,|J|\}, \quad A=A_{|J|}, \quad k_{|J|}=k_{r}+|J|-r .
$$

This together with the equality $\left|S_{r}^{(I)}(A)\right|=|A|-|J|+1$ yields the second part.

Lemma 2. Let $A$ and $B$ be finite subsets of $\mathbb{Z}$ with $4 \leq k=|A|<l=|B|$, $A \subseteq B, \min A=\min B, \max A \neq \max B$ and $|S(A, B)|=k+l-2$. Then $B$ is an $A P$.

Proof. Let $A=\left\{a_{1}, \ldots, a_{k}\right\}$ and $B=\left\{b_{1}, \ldots, b_{l}\right\}$ where $a_{1}<\ldots<a_{k}$ and $b_{1}<\ldots<b_{l}$. Put $C=\left\{a_{1}+b_{2}, \ldots, a_{1}+b_{l-1}, a_{1}+b_{l}, \ldots, a_{k}+b_{l}\right\}$. Clearly $C \subseteq S(A, B)$ and $|C|=k+l-2$. As $|S(A, B)|=k+l-2, S(A, B)$ coincides with $C$. Since $A \subseteq B$ and $a_{k} \neq b_{l}$, for $i=2, \ldots, k$ we may suppose that $a_{i}=b_{f(i)}$ where $i \leq f(i)<l$. Because

$$
\begin{aligned}
S(A, B) \supseteq & \left\{a_{1}+b_{j}: 2 \leq j<f(i)\right\} \cup\left\{a_{i}+b_{j}: j \neq f(i)\right\} \\
& \cup\left\{a_{j}+b_{l}: i<j \leq k\right\},
\end{aligned}
$$

we have

$k+l-2=|S(A, B)| \geq(f(i)-2)+(l-1)+(k-i)=k+l+f(i)-i-3$, i.e. $a_{i} \in\left\{b_{i}, b_{i+1}\right\}$. Observe that $a_{3}<a_{k} \leq b_{l-1}$. Since

$$
a_{1}+b_{l-1}<a_{2}+b_{l-1}<a_{3}+b_{l-1}<a_{3}+b_{l},
$$

$a_{2}+b_{l-1}=a_{1}+b_{l}$ and $a_{3}+b_{l-1}=a_{2}+b_{l}$, it follows that

$$
a_{2}-a_{1}=b_{l}-b_{l-1}=a_{3}-a_{2} .
$$

If $a_{2} \neq b_{2}$, then $a_{2}=b_{3}, a_{3}=b_{4}, b_{2}-b_{1}<a_{2}-b_{1}=a_{3}-a_{2}, a_{1}+b_{3}<$ $b_{2}+a_{2}<a_{3}+b_{1}=a_{1}+b_{4}$; this contradicts the fact $a_{2}+b_{2} \in S(A, B)=C$. So $a_{2}=b_{2}$. As $a_{2}+b_{l-1} \in C$ we must have $a_{2}+b_{l-1}=a_{1}+b_{l}$, similarly $a_{2}+b_{l-2}=a_{1}+b_{l-1}, \ldots, a_{2}+b_{3}=a_{1}+b_{4}$. Thus

$$
b_{l}-b_{l-1}=\ldots=b_{4}-b_{3}=a_{2}-a_{1}=b_{2}-b_{1}=a_{3}-b_{2} .
$$

If $a_{3} \neq b_{3}$, then $a_{3}=b_{4}$ and hence $b_{2}=b_{2}-a_{3}+b_{4}=b_{3}$, which is impossible. So $a_{3}=b_{3}$ and $B$ forms an AP.

3. Proof of Theorem. The case $n=1$ is trivial. Below we let $n \geq 2$ and assume the statement holds for smaller values of $n$.

Put $k_{i}=\left|A_{i}\right|$ for $i=1, \ldots, n$. Set $a=\min \bigcup_{i=1}^{n} A_{i}, I=\{1 \leq i \leq n: a \in$ $\left.A_{i}\right\}, r=\min I$ and $t=\max I$. For $i \in I$ let

$$
A_{i}^{\prime}= \begin{cases}A_{i} \backslash\{a\} & \text { if } i \neq r \\ \{a\} & \text { if } i=r\end{cases}
$$


and for $i \in \bar{I}=\{1, \ldots, n\} \backslash I$ put

$$
A_{i}^{\prime}= \begin{cases}A_{i} \backslash\left\{a_{i}\right\} & \text { if } r<i<t \text { and } i \notin M, \\ A_{i} & \text { otherwise, }\end{cases}
$$

where $a_{i}$ is an arbitrary element of $A_{i}$. Apparently all the $A_{i}^{\prime}$ are finite, nonempty and contained in $\mathbb{Z}$, also $\left|S\left(A_{1}^{\prime}, \ldots, A_{n}^{\prime}\right)\right|=\left|S\left(\left\{A_{i}^{\prime}\right\}_{i \neq r}\right)\right|$. Let $k_{i}^{\prime}=\left|A_{i}^{\prime}\right|$ for $i=1, \ldots, n$. Observe that $k_{i}^{\prime}<k_{j}^{\prime}$ if $1 \leq i<j \leq n$ and $i, j \neq r$. By the induction hypothesis,

$$
\left|S\left(\left\{A_{i}^{\prime}\right\}_{i \neq r}\right)\right| \geq \sum_{i \neq r} k_{i}^{\prime}-\frac{(n-1)(n-1+1)}{2}+1>0 .
$$

Suppose that $\max S\left(\left\{A_{i}^{\prime}\right\}_{i \neq r}\right)=\sum_{i \neq r} a_{i}^{\prime}$ where $\left\{a_{i}^{\prime}\right\}_{i \neq r}$ is an SDR of $\left\{A_{i}^{\prime}\right\}_{i \neq r}$. By Lemma 1 there exists a $J \subseteq\{1, \ldots, n\}$ containing $r$ for which $J \backslash\{r\}=\left\{i \neq r: a_{i}^{\prime} \in A\right\}, \quad S_{r}(A) \subseteq S\left(\left\{A_{i}\right\}_{i=1}^{n}\right), \quad\left|S_{r}(A)\right| \geq k_{r}-r+1$, where $A=\bigcup_{j \in J} A_{j}$ and $S_{r}(A)=\left\{\sum_{i=1}^{n} a_{i}^{\prime}: a_{r}^{\prime} \in A \backslash\left\{a_{i}^{\prime}: i \neq r\right\}\right\}$. As $S\left(A_{1}, \ldots, A_{n}\right) \supseteq S\left(A_{1}^{\prime}, \ldots, A_{n}^{\prime}\right) \cup S_{r}(A)$ and

$$
\max S\left(A_{1}^{\prime}, \ldots, A_{n}^{\prime}\right)=a+\sum_{i \neq r} a_{i}^{\prime}=\min S_{r}(A),
$$

we have

$$
\begin{aligned}
\left|S\left(A_{1}, \ldots, A_{n}\right)\right| & \geq\left|S\left(A_{1}^{\prime}, \ldots, A_{n}^{\prime}\right)\right|+\left|S_{r}(A)\right|-1 \\
& \geq \sum_{i \neq r} k_{i}^{\prime}-\frac{n(n-1)}{2}+1+\left(k_{r}-r+1\right)-1 \\
& \geq \sum_{i \neq r} k_{i}-(t-r)-\frac{n(n-1)}{2}+1+k_{r}-r \\
& =\sum_{i=1}^{n} k_{i}-\frac{n(n+1)}{2}+n-t+1 \\
& \geq \sum_{i=1}^{n} k_{i}-\frac{n(n+1)}{2}+1 .
\end{aligned}
$$

From now on we assume that

$$
\left|S\left(A_{1}, \ldots, A_{n}\right)\right|=\sum_{i=1}^{n} k_{i}-\frac{n(n+1)}{2}+1 .
$$

The above deduction yields

$$
\begin{gathered}
\left|S\left(\left\{A_{i}^{\prime}\right\}_{i \neq r}\right)\right|=\sum_{i \neq r} k_{i}-(t-r)-\frac{n(n-1)}{2}+1, \\
r<i<t \Rightarrow i \notin \bar{I} \cap M,
\end{gathered}
$$




$$
\begin{aligned}
\left|S_{r}(A)\right| & =k_{r}-r+1, \\
t & =n .
\end{aligned}
$$

By (13) and Lemma 1 there is an $l \in\{r, \ldots, n\}$ for which $J=\{1, \ldots, l\}$, $\bigcup_{j=1}^{l} A_{j}=A_{l}, k_{l}=k_{r}+(l-r)$ and

$$
\{1, \ldots, l\} \backslash\{r\}=J \backslash\{r\}=\left\{i \neq r: a_{i}^{\prime} \in A=A_{l}\right\} .
$$

(14) indicates that $a \in A_{n}$. Let $b=\max \bigcup_{i=1}^{n} A_{i}$. Clearly $a \neq b$, for otherwise each $A_{i}$ contains exactly one element, which contradicts the inequality $k_{1}<$ $k_{n}$. As $-b=\min \bigcup_{i=1}^{n}\left(-A_{i}\right)$ and $\left|S\left(-A_{1}, \ldots,-A_{n}\right)\right|=\left|S\left(A_{1}, \ldots, A_{n}\right)\right|=$ $\sum_{i=1}^{n}\left|-A_{i}\right|-n(n+1) / 2+1$, similarly we have $-b \in-A_{n}$. So $b \in A_{n}^{\prime}=$ $A_{n} \backslash\{a\}$. Choose the smallest $s \leq n$ such that $b \in A_{s}$.

Let $m \in M$. We now show that $\bigcup_{i=1}^{m} A_{i}=A_{m}$, i.e. $A_{m}$ contains both $\bigcup_{i=1, i \neq r}^{m} A_{i}$ and $A_{r}$.

If $m=r$, then $r \in M$, hence $l=r=m$ and $A_{m}=\bigcup_{i=1}^{m} A_{i} \supseteq$ $\bigcup_{i=1, i \neq r}^{m} A_{i}$.

Since $A_{i}^{\prime}=A_{i}$ for all $i<r$, by (11), (12) and the induction hypothesis, if $m<r$ then $\bigcup_{i=1}^{m} A_{i}=\bigcup_{i=1, i \neq r}^{m} A_{i}=A_{m}$.

Assume $r<n$. Clearly $b \in\left\{a_{i}^{\prime}: i \neq r\right\}$ (otherwise $\sum_{i \neq r, n} a_{i}^{\prime}+b \in$ $S\left(\left\{A_{i}^{\prime}\right\}_{i \neq r}\right)$ would be greater than $\left.\sum_{i \neq r} a_{i}^{\prime}=\max S\left(\left\{A_{i}^{\prime}\right\}_{i \neq r}\right)\right)$. Suppose that $b=a_{j}^{\prime}$ where $j \neq r$. In view of $(15), b \in A_{l}$ if and only if $j \leq l$. Since $b=a_{j}^{\prime} \in A_{j}^{\prime} \subseteq A_{j}$, we have $j \geq s$. If $l=s$, then $b \in A_{l}, s \leq j \leq l=s$, $s=j \neq r$.

Now suppose that $r<m \leq n$. If $m<t=n$, then $m \in I$ by (12), and $k_{m+1}^{\prime}-k_{m}^{\prime} \geq\left(k_{m+1}-1\right)-\left(k_{m}-1\right)>1$. By (11), (12) and the induction hypothesis $\bigcup_{i=1, i \neq r}^{m} A_{i}^{\prime}=A_{m}^{\prime}$. If $1 \leq i<r$, then $A_{i}=A_{i}^{\prime} \subseteq A_{m}^{\prime} \subseteq A_{m}$; if $r<i \leq m$ and $i \in I$, then $A_{i}=A_{i}^{\prime} \cup\{a\} \subseteq A_{m}^{\prime} \cup\{a\}=A_{m}$; if $r<i \leq m$ but $i \notin I$, then $\left|A_{i}\right| \geq k_{r}+1 \geq 2$ and hence for any given $x_{i} \in A_{i}$ by taking $a_{i} \in A_{i}$ different from $x_{i}$ at the beginning we find that $x_{i} \in A_{i} \backslash\left\{a_{i}\right\}=A_{i}^{\prime} \subseteq A_{m}^{\prime} \subseteq A_{m}$. So $\bigcup_{i=1, i \neq r}^{m} A_{i} \subseteq A_{m}$.

Since $s$ is the smallest index such that $-A_{s}$ contains $\min \bigcup_{i=1}^{n}\left(-A_{i}\right)=$ $-b$, by analogy $\bigcup_{i=1, i \neq s}^{m}\left(-A_{i}\right) \subseteq-A_{m}$. Thus, if $r \neq s$ then $-A_{r} \subseteq-A_{m}$, i.e. $A_{r} \subseteq A_{m}$. If $r=s$, then by the above $l \neq s=r$, also $l \leq m$ since $k_{l}=k_{r}+l-r$, therefore $A_{r} \subseteq \bigcup_{j=1}^{l} A_{j}=A_{l} \subseteq A_{m}$. So $\bigcup_{i=1}^{m} A_{i}=A_{m}$.

Now let us check that $A_{n}$ is an AP except the case $k_{1} \leq 3$.

If $r=1$ then $\min \left\{k_{i}^{\prime}: i \neq r\right\}=k_{2}^{\prime} \geq k_{2}-1 \geq k_{1}$, and if $r>1$ then $\min \left\{k_{i}^{\prime}: i \neq r\right\}=k_{1}^{\prime}=k_{1}$. So $\min \left\{k_{i}^{\prime}: i \neq r\right\} \geq k_{1}$. Below we assume that $k_{1} \geq 4$.

Suppose $n>2$. By (11), (12) and the induction hypothesis, if $r<n$ then $A_{n}^{\prime}=A_{n} \backslash\{a\}$ is an AP. Similarly, if $s<n$ then $-A_{n} \backslash\{-b\}$ is an AP and hence so is $A_{n} \backslash\{b\}$. Thus, if $r<n$ and $s<n$ then $A_{n}=\{a\} \cup\left(A_{n} \backslash\{a\}\right)=$ $\left(A_{n} \backslash\{b\}\right) \cup\{b\}$ forms an AP. (Note that $\left|A_{n}\right|=k_{n}>k_{1} \geq 4$.) 
Now consider the case $r=s=1<n=2$. By the above $l=2$ (since $l \neq s=1$ ) and $k_{2}=k_{1}+1$. Let $a=b_{1}<\ldots<b_{k_{2}}=b$ be all the elements of $A_{2}$. If $1 \leq i<j \leq k_{2}$, then either $b_{i}$ or $b_{j}$ belongs to $A_{1}$ because $\left|A_{2} \backslash A_{1}\right|=1$, therefore $b_{i}+b_{j} \in S\left(A_{1}, A_{2}\right)$. So

$$
\begin{aligned}
S\left(A_{1}, A_{2}\right) & \supseteq S\left(A_{2} \backslash\{b\}, A_{2}\right) \\
& \supseteq C=\left\{b_{1}+b_{2}, \ldots, b_{1}+b_{k_{2}}, b_{2}+b_{k_{2}}, \ldots, b_{k_{2}-1}+b_{k_{2}}\right\} .
\end{aligned}
$$

As $\left|S\left(A_{1}, A_{2}\right)\right|=k_{1}+k_{2}-2=2 k_{2}-3=|C|$, we have $S\left(A_{1}, A_{2}\right)=C=$ $S\left(A_{2} \backslash\{b\}, A_{2}\right)$. Clearly $\left|A_{2} \backslash\{b\}\right|=k_{2}-1=k_{1} \geq 4, \min \left(A_{2} \backslash\{b\}\right)=\min A_{2}$ and $\max \left(A_{2} \backslash\{b\}\right) \neq \max A_{2}$. Applying Lemma 2 we find that $A_{n}=A_{2}$ forms an AP.

With respect to the case $r=n$ we make the following remarks:

(i) Since $A_{n}=\bigcup_{j=1}^{n} A_{j}$, we have $A_{n-1} \subset A_{n}$. If $n>2$, then by (11), (12) and the induction hypothesis $A_{n-1}^{\prime}=\bigcup_{i=1}^{n-1} A_{i}^{\prime}$ forms an AP, i.e. $\bigcup_{i=1}^{n-1} A_{i}=$ $A_{n-1}$ is an AP. Set

$$
A_{n}^{-}=\left\{x \in A_{n}: x \leq \max A_{n-1}\right\} \quad \text { and } \quad A_{n}^{+}=\left\{x \in A_{n}: \min A_{n-1} \leq x\right\} .
$$

Whether $n=2$ or $n>2$ we always have $\bigcup_{i=1}^{n-1} A_{i}=A_{n-1} \subseteq A_{n}^{-} \cap A_{n}^{+}$and hence $\left|A_{n}^{-} \cap A_{n}^{+}\right| \geq k_{n-1} \geq k_{1} \geq 4$. Among $A_{1}, \ldots, A_{n-1}, A_{n}^{-}$, the index $r^{-}$ of the first one containing $\min \left(\bigcup_{i=1}^{n-1} A_{i} \cup A_{n}^{-}\right)=a$ is identical with $r$ while the index $s^{-}$of the first one containing $\max \left(\bigcup_{i=1}^{n-1} A_{i} \cup A_{n}^{-}\right)=\max A_{n-1}$ is less than $n$. Similarly, among $A_{1}, \ldots, A_{n-1}, A_{n}^{+}$, the index $r^{+}$of the first one containing $\min \left(\bigcup_{i=1}^{n-1} A_{i} \cup A_{n}^{+}\right)=\min A_{n-1}$ is less than $n$ while the index $s^{+}$of the first one containing $\max \left(\bigcup_{i=1}^{n-1} A_{i} \cup A_{n}^{+}\right)=b$ is equal to $s$.

(ii) Suppose that $A_{n}^{-} \neq A_{n-1}$. Then $\left|A_{n}^{-}\right|>k_{n-1}>\ldots>k_{1}$. According to the previous reasoning,

$$
\left|S\left(A_{1}, \ldots, A_{n-1}, A_{n}^{-}\right)\right| \geq \sum_{i=1}^{n-1} k_{i}+\left|A_{n}^{-}\right|-\frac{n(n+1)}{2}+1>0 .
$$

Observe that

$$
\begin{aligned}
\max S\left(A_{1}, \ldots, A_{n-1}, A_{n}^{-}\right) & \leq \max S\left(A_{1}^{\prime}, \ldots, A_{n-1}^{\prime}\right)+\max A_{n-1} \\
& <\max S\left(\left\{a_{1}^{\prime}\right\}, \ldots,\left\{a_{n-1}^{\prime}\right\}, A_{n} \backslash A_{n}^{-}\right),
\end{aligned}
$$

and that $\left|S\left(\left\{a_{1}^{\prime}\right\}, \ldots,\left\{a_{n-1}^{\prime}\right\}, A_{n} \backslash A_{n}^{-}\right)\right|=\left|A_{n} \backslash A_{n}^{-}\right|$. So

$$
\begin{aligned}
\left|S\left(A_{1}, \ldots, A_{n}\right)\right| & \geq\left|S\left(A_{1}, \ldots, A_{n-1}, A_{n}^{-}\right)\right|+\left|S\left(\left\{a_{1}^{\prime}\right\}, \ldots,\left\{a_{n-1}^{\prime}\right\}, A_{n} \backslash A_{n}^{-}\right)\right| \\
& \geq \sum_{i=1}^{n-1} k_{i}+\left|A_{n}^{-}\right|-\frac{n(n+1)}{2}+1+\left(k_{n}-\left|A_{n}^{-}\right|\right) \\
& =\sum_{i=1}^{n} k_{i}-\frac{n(n+1)}{2}+1 .
\end{aligned}
$$


Since (10) holds, we must have

$$
\left|S\left(A_{1}, \ldots, A_{n-1}, A_{n}^{-}\right)\right|=\sum_{i=1}^{n-1} k_{i}+\left|A_{n}^{-}\right|-\frac{n(n+1)}{2}+1 .
$$

(iii) By analogy, when $-A_{n}^{+}=\left\{-x \in-A_{n}:-x \leq \max \left(-A_{n-1}\right)\right\} \neq$ $-A_{n-1}$ (i.e. $A_{n}^{+} \neq A_{n-1}$ ), we have

$$
\begin{aligned}
\left|S\left(A_{1}, \ldots, A_{n-1}, A_{n}^{+}\right)\right| & =\left|S\left(-A_{1}, \ldots,-A_{n-1},-A_{n}^{+}\right)\right| \\
& =\sum_{i=1}^{n-1} k_{i}+\left|A_{n}^{+}\right|-\frac{n(n+1)}{2}+1 .
\end{aligned}
$$

Assume that $s<r=n$. Then both $r^{+}$and $s^{+}=s$ are less than $n$. If $A_{n}^{+} \neq A_{n-1}$, then (17) holds and hence $A_{n}^{+}$forms an AP by previous arguments. If $n>2$, then $A_{n}^{+}$is an AP anyway and so is $A_{n} \backslash\{b\}$ by the above, therefore $A_{n}$ forms an AP. If $n=2$, then $s=1, \min \left(-A_{1}\right)=$ $\min \left(-A_{2}\right), \max \left(-A_{1}\right) \neq \max \left(-A_{2}\right)$ (since $\left.r=2\right)$, and $\left|S\left(-A_{1},-A_{2}\right)\right|=$ $\left|S\left(A_{1}, A_{2}\right)\right|=k_{1}+k_{2}-2$, hence $-A_{2}$ is an AP by Lemma 2 , thus $A_{n}=A_{2}$ forms an AP.

In the case $r<s=n$, by applying the above result to the subsets $-A_{1}, \ldots,-A_{n}$ instead of $A_{1}, \ldots, A_{n}$, we see that $-A_{n}$ forms an AP, i.e., $A_{n}$ is an AP.

Finally, we handle the remaining case $r=s=n$. Since $r^{+}<s^{+}=s=n$ and $s^{-}<r^{-}=r=n$, by the above $A_{n}^{+}$forms an AP if $A_{n}^{+} \neq A_{n-1}$, and $A_{n}^{-}$forms an $\mathrm{AP}$ if $A_{n}^{-} \neq A_{n-1}$. Providing $n>2$, both $A_{n}^{+}$and $A_{n}^{-}$are APs, therefore $A_{n}$ forms an AP. When $n=2$, if $A_{n}=A_{2}$ is not an AP, then $A_{2}^{-}$or $A_{2}^{+}$coincides with $A_{1}$, hence $\min A_{1}=\min A_{2}^{-}=\min A_{2}$ and $\max A_{1} \neq \max A_{2}$ (since $\left.s=2\right)$, or $\min \left(-A_{1}\right)=\min \left(-A_{2}^{+}\right)=\min \left(-A_{2}\right)$ and $\max \left(-A_{1}\right) \neq \max \left(-A_{2}\right)$ (since $r=2$ ), thus $A_{2}$ forms an AP by Lemma 2 , which leads a contradiction. So, whether $n>2$ or $n=2, A_{n}$ always forms an AP.

The induction step is now completed and the proof of the Theorem is finished.

\section{References}

[ANR1] N. Alon, M. B. Nathanson and I. Z. Ruzsa, Adding distinct congruence classes modulo a prime, Amer. Math. Monthly 102 (1995), 250-255.

[ANR2] - - - - , The polynomial method and restricted sums of congruence classes, J. Number Theory 56 (1996), 404-417.

[B] Y. Bilu, Addition of sets of integers of positive density, ibid. 64 (1997), 233-275.

[DH] J. A. Dias da Silva and Y. O. Hamidoune, Cyclic space for Grassmann derivatives and additive theory, Bull. London Math. Soc. 26 (1994), 140-146. 
[EH] P. Erdős and H. Heilbronn, On the addition of residue classes mod $p$, Acta Arith. 9 (1964), 149-159.

[G] R. K. Guy, Unsolved Problems in Number Theory, 2nd ed., Springer, New York, 1994, 129-131.

[H] P. Hall, On representatives of subsets, J. London Math. Soc. 10 (1935), 26-30.

$[\mathrm{J}] \quad$ N. Jacobson, Basic Algebra I, 2nd ed., W. H. Freeman and Company, New York, 1985.

[N] M. B. Nathanson, Inverse theorems for subset sums, Trans. Amer. Math. Soc. 347 (1995), 1409-1418.

Hui-Qin Cao

The Fundamental Division

Nanjing Auditing College

Nanjing 210029

The People's Republic of China
Zhi-Wei Sun

Department of Mathematics Nanjing University Nanjing 210093

The People's Republic of China E-mail: zwsun@netra.nju.edu.cn

Received on 1.3.1998

and in revised form on 9.8.1998 